\title{
Costos comparativos entre laparoscopia ginecológica ambulatoria y Cirugía ginecológica por laparotomía
}

\author{
Ramiro Argüello Argüello*
}

\section{RESUMEN}

Se comparan costos entre procedimientos ginecológicos que pueden realizarse por laparoscopia operatoria ambulatoria, o por laparotomía. Se utilizaron las tarifas del Instituto de Seguro Social de 1.999, por ser las más utilizadas a nivel nacional. En todos los procedimientos, a excepción de uno, fue mayor el costo $(0,7 \%$ al $55,7 \%)$ al realizarlo por laparotomía. Se analizan las causas de disminución de costos de la laparoscopia en Colombia. Se concluye que en nuestro país la primera opción quirúrgica, para la mayoría de la patología benigna ginecológica intraabdominal, debe ser la laparoscopia ambulatoria, realizada por ginecólogos entrenados y en condiciones adecuadas.

PALABRAS CLAVES: Costos, Laparoscopia, Laparotomía, Endoscopia Ginecológica, Colombia.

\section{SUMMARY}

Costs among gynecological procedures that can be made by ambulatory laparoscopy or by laparotomy are compared. Instituto de Seguro Social's fares (1.999) were used because they are de most used at national level. In all procedures, except one, laparotomy's cost was higher $(0,7 \%$ to $55,7 \%)$. The causes for the lower in costs of laparoscopy in Colombia are explained. So, in Colombia the first surgical choice for most of the benign abdominal gynecological pathologies must be ambulatory laparoscopy, when it is made by a well trained gynecology, and performed in good conditions.

KEY WORDS: Cost studies, Laparoscopy, Laparotomy, Gynecological Endoscopy, Colombia.

\section{Introducción}

En Colombia y en el mundo se vienen imponiendo los procedimientos menos invasivos frente a los considerados como de cirugía tradicional. Es así como la cirugía por video o cirugía de invasión mínima se vuelve cada vez más frecuente en las especialidades quirúrgicas (1).

En Ginecología esta tendencia es muy notoria, a tal punto que hoy día un gran número de los procedimientos que anteriormente se debían realizar mediante laparotomía se hacen ahora, preferiblemente, de manera ambulatoria por videolaparoscopia (2). Incluso Gomel anota que ya es menor el número de los procedimientos ginecológicos que no pueden hacerse mediante laparoscopia, que aquellos que sí (3).

Actualmente, las LAPAROSCOPIAS GINECOLOGICAS OPERATORIAS se encuentran incluidas en los manuales de tarifas del Instituto de Seguros Sociales (I.S.S.) y del Seguro Obligatorio de Accidentes de Tránsito (S.O.A.T.). Los diferentes Niveles de Cirugía

Coordinador de la Comisión de Certificación en Endoscopia Ginecológica de la Sociedad Colombiana de Obstetricia y Ginecología. Certificación de Experto en Endoscopia Ginecológica dado por la Sociedad Colombiana de Obstetricia y Ginecología. Director Científico de la Unidad de Endoscopia Ginecológica y Dolor Pélvico. UNEGIN LTDA.
Laparoscópica contemplados en estos Manuales de Tarifas ya cuentan con más del $60 \%$ a $70 \%$ de los procedimientos que anteriormente solo se hacían mediante Laparotomía (4,5). Es muy importante resaltar que al efectuar estas cirugías mediante Laparoscopia se están convirtiendo, en su mayoría, en CIRUGIAS AMBULATORIAS, con ventajas innegables para las pacientes, permitiendo al mismo tiempo un mejor manejo de las camas hospitalarias en las instituciones.

Por lo tanto es muy importante determinar si en nuestro medio la CIRUGIA GINECOLOGICA LAPAROSCOPICA AMBULATORIA implica también ventajas desde el punto de vista de costos, comparada con la LAPAROTOMIA, ya que dentro de las modificaciones que ha traído la Ley 100 en Colombia en el área de la Salud, uno de los aspectos a los cuales se le está prestando cada vez mayor atención es el relacionado con los costos generados por los diferentes procedimientos quirúrgicos.

En este sentido, no son aplicables las tarifas médicas o estudios de costos desarrollados en otros países $(6,7,8,9)$, ya que en Colombia se utilizan mayoritariamente los Manuales Tarifarios mencionados, los cuales tienen especificaciones y limitantes solo aplicables en nuestras instituciones. Se impone entonces demostrar en nuestro medio, y con estos manuales, la ventaja o desventaja desde el punto de vista económico de la Cirugía Ginecológica 
Laparoscópica Ambulatoria. Los beneficios para las pacientes en todos los demás aspectos ya están suficientemente demostrados y aceptados a nivel mundial $(2,7,9)$.

\section{Materiales y Métodos}

Se utilizó el Manual de Procedimientos y tarifas del Instituto de Seguros Sociales (I.S.S.), vigente en 1.999, por considerar que es uno de los más utilizados a nivel nacional, y más completo desde el punto de vista de la Endoscopia Ginecológica (4).

Los procedimientos comparados fueron aquellos que pueden realizarse, rutinariamente, bien sea por LAPAROSCOPIA OPERATORIA AMBULATORIA O por LAPAROTOMÍA, y se encuentran claramente especificados en el Manual de tarifas del I.S.S..

Para efectos de este estudio de costos también se incluyeron procedimientos que no tienen igual denominación por laparotomía y laparoscopia, pero tienen objetivos terapéuticos similares. Estos son la fulguración de ovarios por laparoscopia con la cuña de ovarios por laparotomía; al igual que la sección laparoscópica de adherencias a pared abdominal con la liberación de adherencias peritoneales por laparotomía.

Los procedimientos comparados y sus correspondientes Unidades de Valor Relativo (U.V.R.) se describen en la Tabla No. 1.

No fue posible comparar la Sección laparoscópica de ligamentos utero sacros, ni el manejo laparoscópico de la endometriosis, ya que no tienen contraparte especifica mediante laparotomía.

Todos los procedimientos se facturaron por actividad (o evento), y no bajo la modalidad de Conjuntos de Atención en Salud por Tarifa Integral ("paquete").
Para calcular los costos no estipulados estrictamente en el Manual, (costos variables dados principalmente por medicamentos y materiales que se facturan independientemente), se promediaron los valores de estos elementos utilizados en cada intervención analizada. Para calcular estos promedios se estudiaron los costos variables de las últimas 50 laparoscopias operatorias ambulatorias, y de igual número de las diferentes cirugías ginecológicas por laparotomía, realizadas en nuestra Unidad en los últimos 12 meses, actualizando costos a la fecha. Ninguno de los procedimientos analizados presentó complicaciones.

Se encontró que hay muy poca variación en la utilización de estos elementos y medicamentos en una laparoscopia operatoria ambulatoria, sin importar el tipo del procedimiento practicado. Igual resultado se encontró en la cirugía ginecológica por laparotomía, razón por la cual se tomó un promedio para Laparoscopia Operatoria Ambulatoria y otro para Cirugía ginecológica por Laparotomía.

La estancia promedio para una cirugía por laparotomía se consideró de dos (2) días, realizada en una Institución de II Nivel de complejidad, con habitaciones bipersonales.

Se consideró que en el $100 \%$ de los casos se requirió estudio anatomopatológico.

La incapacidad causada para la Cirugía laparoscópica ambulatoria normalmente está entre 5 y 8 días y se tomó un promedio de siete (7) días para los casos analizados. La incapacidad promedio para la laparotomía está entre 20 y 30 días y se tomaron 25 días como promedio para la Laparotomía.

Para el cálculo del valor de la incapacidad laboral se utilizó el salario mínimo mensual vigente en Colombia, el cual es de $\$ 236.460$ (\$7.882 día).

Tabla 1

\begin{tabular}{|c|c|c|}
\hline PROCEDIMIENTO & LAPAROSCOPIA & LAPAROTOM \\
\hline EmbarazoEctópico & 90 U.V.R. & 100 U.V.R. \\
\hline Cistectomía & 110 U.V.R. & 100 U.V.R. \\
\hline Cistectomía (Quiste intraligamentario) & 110 U.V.R. & 110 U.V.R. \\
\hline Miomectomía (mioma pediculado) & 100 U.V.R. & 100 U.V.R. \\
\hline Miomectomía (mioma subseroso) & 120 U.V.R. & 100 U.V.R. \\
\hline Salpingectomía y/o ooforectomía & 120 U.V.R. & 120 U.V.R. \\
\hline Salpingoplastia & 120 U.V.R. & 125 U.V.R. \\
\hline Salpingolisis (Adherenciacias leves) & 110 U.V.R. & 90 U.V.R. \\
\hline Salpingolisis (Adh. Moderadas a severas) & 140 U.V.R. & 90 U.V.R. \\
\hline Extracción D.I.U. Intraabdominal & 70 U.V.R. & 60 U.V.R. \\
\hline Histerorrafia & 80 U.V.R. & 80 U.V.R. \\
\hline Fulguración ovarios Vs. Cuña ovarios & 90 U.V.R. & $6 \dot{5}$ U.V.R. \\
\hline Adherencias Pared Abdominal Vs. Peritoneales & 70 U.V.R. & 140 U.V.R. \\
\hline
\end{tabular}




\section{Resultados}

En las Tablas 2 y 3 se pueden apreciar los costos totales, con base en las U.V.R. generadas por las intervenciones de Cirugía Laparoscópica Ambulatoria y las correspondientes, realizadas por Laparotomía.

En la Tabla 4 se comparan los costos totales obtenidos para cada procedimiento, bien sea por laparoscopia o por laparotomía. Se puede apreciar que al realizar un procedimiento por laparotomía y no por laparoscopia, se generan sobrecostos que pueden ir del $0,7 \%$ al $55,7 \%$. El único caso en el que se generó un mayor costo $(10,2 \%)$ al realizar el procedimiento por laparoscopia fue el de la salpingolisis de adherencias moderadas o severas.

\section{Discusión}

En Colombia algunas entidades de salud no autorizan con facilidad los procedimientos laparoscópicos operatorios en ginecología. Posiblemente piensan estar disminuyendo costos al autorizar únicamente intervenciones por Laparotomía y no por Laparoscopia.

Tabla 2 (Laparoscopia)

\begin{tabular}{|c|c|c|c|c|c|c|c|}
\hline U.V.R. & 70 & 80 & 90) & 100 & 110 & 120 & 140 \\
\hline Consulta Preanestésica & 11,350 & 11,350 & 11,350 & 11,350 & 11,350 & 11,350 & 11,350 \\
\hline Consulta Prequirúrgica & 11,350 & 11,350 & 11,350 & 11,350 & 11,350 & 11,350 & 11,350 \\
\hline Honorarios Anestesia & 64,050 & 73,200 & 82,350 & 91,500 & 100,650 & 109,800 & 128,100 \\
\hline Honorarios Cirujano & 84,700 & 96,800 & 108,900 & 121,000 & 133,100 & 145,200 & 169,400 \\
\hline Honorarios Ayudante & 24.150 & 27,600 & 31,050 & 34.500 & 37,950 & 41,400 & 48,306 \\
\hline Derechos de Sala & 79,935 & 95,095 & 107,375 & 119,790 & 123,020 & 126,770 & 154,375 \\
\hline Drogas (promedio) & 31,575 & 31,575 & 31,575 & 31,575 & 31,575 & 31,575 & 31,575 \\
\hline \multicolumn{8}{|l|}{ Trocar desechable $1^{a}$. } \\
\hline Punción & 263,200 & 263,200 & 263,200 & 263,200 & 263,200 & 263,200 & 263,200 \\
\hline Material Quirúrgico & 71,265 & 76,720 & 82,260 & 94,550 & 106,760 & 113,520 & 121,315 \\
\hline Patología (Cod. 20201) & 26,450 & 26,450 & 26,450 & 26.450 & 26,450 & 26,450 & 26,450 \\
\hline Incapacidad & 55,174 & 55,174 & 55,174 & 55,174 & 55,174 & 55,174 & 55,174 \\
\hline VALOR TOTAL & 723,199 & 768,514 & 811,034 & 860,439 & 900,579 & 935,789 & $1,020,58$ \\
\hline
\end{tabular}

Tabla 3 (Laparotomía)

\begin{tabular}{|lrrrrrrrrr|}
\hline U.V.R. & $\mathbf{6 0}$ & $\mathbf{6 5}$ & $\mathbf{8 0}$ & $\mathbf{9 0}$ & $\mathbf{1 0 0}$ & $\mathbf{1 1 0}$ & $\mathbf{1 2 0}$ & $\mathbf{1 2 5}$ & $\mathbf{1 4 0}$ \\
\hline Consulta Preanestésica & 11,350 & 11,350 & 11,350 & 11,350 & 11,350 & 11,350 & 11,350 & 11,350 & 11,350 \\
Consulta Prequirúrgica & 11,350 & 11,350 & 11,350 & 11,350 & 11,350 & 11,350 & 11,350 & 11,350 & 11,350 \\
Honorarios Anestesia & 54,900 & 59,475 & 73,200 & 82,350 & 91,500 & 100,650 & 109,800 & 114,375 & 128,100 \\
Honorarios Cirujano & 72,600 & 78,650 & 96,800 & 108,900 & 121,000 & 133,100 & 145,200 & 151,250 & 169,400 \\
Honorarios Ayudante & 20,700 & 22,425 & 27,600 & 31,050 & 34,500 & 37,950 & 41,400 & 43,125 & 48,300 \\
Derechos de Sala & 67.225 & 79,935 & 95,095 & 107,375 & 119,790 & 123,020 & 126,770 & 126,770 & 154,375 \\
Drogas (promedio) & 147,063 & 147,063 & 147,063 & 147,063 & 147,063 & 147,063 & 147,063 & 147,063 & 147,063 \\
Material Quirúrgico & 49,705 & 71,265 & 76,720 & 82,260 & 94,550 & 106,760 & 113,520 & 113,520 & 121,315 \\
Patología (Cod. 20201) & 26,450 & 26,450 & 26,450 & 26,450 & 26,450 & 26,450 & 26,450 & 26,450 & 26,450 \\
Habitación dos días & & & & & & & 11,450 & \\
(Bipersonal Nivel II) & 111,380 & 111,380 & 111,380 & 111,380 & 111,380 & 111,380 & 111,380 & 111,380 & 111,380 \\
Incapacidad & 197,050 & 197,050 & 197,050 & 197,050 & 197,050 & 197,050 & 197,050 & 197,050 & 197,050 \\
\hline VALOR TOTAL & $\mathbf{7 6 9 , 7 7 3}$ & $\mathbf{8 1 6 , 3 9 3}$ & $\mathbf{8 7 4 , 0 5 8}$ & $\mathbf{9 1 6 , 5 7 8}$ & $\mathbf{9 6 5 , 9 8 3}$ & $\mathbf{1 , 0 0 6 , 1 2 3}$ & $\mathbf{1 , 0 4 1 , 3 3 3}$ & $\mathbf{1 , 0 5 3 , 6 8 3}$ & $\mathbf{1 , 1 2 6 , 1 3 3}$ \\
\hline
\end{tabular}


Tabla 4

\begin{tabular}{|c|c|c|c|c|c|}
\hline PROCEDIMIENTO & $\begin{array}{c}\text { Costo } \\
\text { Laparoscopia }\end{array}$ & $\begin{array}{c}\text { Costo } \\
\text { Laparotomía }\end{array}$ & $\begin{array}{c}\text { Sobrecosto } \\
\text { Laparotomía }\end{array}$ & $\begin{array}{l}\text { Sobrecosto } \\
\text { Laparoscopia }\end{array}$ & $\begin{array}{l}\text { Porcentaje } \\
\text { sobrecosto }\end{array}$ \\
\hline Embarazo Ectópico & 811,034 & 965,983 & 154,949 & & 19.1 \\
\hline Cistectomía & 900,579 & 965,983 & 65,404 & & 7.3 \\
\hline Cistectomía (Quiste Intraligamentario) & 900,579 & $1,006,123$ & 105,544 & & 11.7 \\
\hline Miomectomía (Mioma Pediculado) & 860,439 & 965,983 & 105,544 & & 12.3 \\
\hline Miomectomía (Mioma Subseroso) & 935,789 & $1,041,333$ & 105,544 & & 11.3 \\
\hline Salpingectomía y/o Ooforectomía & 935,789 & $1,041,333$ & 105,544 & & 11.3 \\
\hline Salpingoplastia & 935,789 & $1,053,683$ & 117,894 & & 12.6 \\
\hline Salpingolisis (Adherencias Leves) & 900,579 & 916,578 & 15,999 & & 1.8 \\
\hline Salpingolisis (Adh. Moderadas a Severas) & $1,020,589$ & 916,578 & & 104,011 & 10.2 \\
\hline Extracción D.I.U. Intraabdominal & 723,199 & 769,773 & 46,574 & & 6.4 \\
\hline Histerorrafia & 768,514 & 874,058 & 105.544 & & 13.7 \\
\hline FuIguración Ovarios Vs. Cuña Ovarios & 811,034 & 816,393 & 5,359 & & 0.7 \\
\hline Adher. Pared Abd. Vs Adher. Peritoneales & 723,199 & $1,126,133$ & 402,934 & & 55.7 \\
\hline
\end{tabular}

Los costos aquí mostrados indican claramente que cuando se tiene en cuenta la incapacidad laboral, la primera opción, para la mayoría de los procedimientos, está a favor de la Cirugía ambulatoria por Laparoscopia. Esto es mucho más obvio con pacientes que tengan ingresos superiores al salario mínimo aquí utilizado, ya que el pago por cada incapacidad es mayor.

Tal vez la razón por la cual algunas personas aún creen que la Laparoscopia Operatoria, en ginecología, es más costosa que la cirugía convencional por Laparotomía, pueda encontrarse en la historia del desarrollo de este tipo de cirugía en nuestro medio, antes y después de la utilización de las técnicas de video.

Cuando se introdujo la cirugía por Videolaparoscopia en Colombia, la experiencia en laparoscopia ginecológica (sin técnicas de video) era muy amplia (10). Sin embargo, la novedad de la introducción de la entonces llamada "cirugía de invasión mínima" destacó principalmente la Colecistectomía por Laparoscopia (11) y después, en ginecología, se hizo mucho énfasis en la Histerectomía por Laparoscopia. Este no fue un buen comienzo en Endoscopia Ginecológica ya que la histerectomía por laparoscopia es una cirugía que requiere gran experiencia y, aún hoy en día, genera controversia (12). Es lógico que en esta etapa los costos de los procedimientos fueran elevados, principalmente por los siguientes factores:

a) Para iniciar este tipo de cirugía las instituciones debían adquirir la totalidad de los equipos, con precios no regulados por una adecuada oferta. Además no había claridad, sobre todo en ginecología, sobre el instrumental específico requerido, lo que ocasionaba compras innecesarias. Lógicamente muchos de estos costos de compra se transladaban al procedimiento, con el fin de recuperar la inversión inicial. b) Se utilizaban indiscriminadamente, sin mayor criterio de selección, muchos elementos desechables en cada cirugía. Este fenómeno se explica principalmente por la presión de las casas productoras, ansiosas de crear un mercado para sus productos. Obviamente los costos de cada procedimiento podían hasta triplicarse solo por el valor de los elementos desechables utilizados. Debe recordarse que llegaron a ofrecerse hasta costosas ópticas desechables y que en la Histerectomía por Laparoscopia se recomendaba utilizar profusamente instrumental desechable y suturas mecánicas.

c) El inicio de la curva de entrenamiento de cirujanos y ginecólogos hacía que el tiempo quirúrgico fuera mayor, lo que ocasionaba mayor consumo de drogas anestésicas, derechos de sala, etc.

Actualmente en Colombia los costos han bajado porque los equipos se encuentran en casi todo el territorio Colombiano y las instituciones de salud solo deben preocuparse por gastos de reposición. En ginecología se reglamentaron las necesidades específicas en equipo e instrumental (13), evitando así compras superfluas. Además, los elementos desechables se han restringido al máximo, a tal punto que en ginecología solo se contempla el trocar desechable para primera punción como único elemento desechable de uso rutinario (4), indispensable si se desea disminuir al máximo la lesión de grandes vasos. Se encuentran ginecólogos experimentados y certificados que ya han completado su curva de entrenamiento (14), y en muchos casos operan por parejas, disminuyendo tiempos quirúrgicos y complicaciones. Su misma experiencia les permite resistir la presión de las casas productoras, evaluando con ojo crítico las nuevas tecnologías u ofertas presentadas.

Una estrategia recomendable es el equiparar costos entre la cirugía ginecológica por laparotomía o por 
laparoscopia. Se evita así la tendencia de anteponer el estimativo de los costos a la conveniencia de la paciente.

En Colombia, en el área de la Endoscopia Ginecológica, ya se dio un primer paso para lograr el objetivo de alcanzar costos similares, independientemente de la técnica quirúrgica utilizada. Esto se puede apreciar en el Manual de Procedimientos y Tarifas del I.S.S. para 1999, donde se incluye un Conjunto de Atención en Salud por Tarifa Integral ("Paquete") para la LAPAROTOMIA GINECOLOGICA (4). El costo total de este conjunto es muy similar al de los Conjuntos de Cirugía Ginecológica Laparoscópica Ambulatoria.

Otra posible razón para que algunas entidades de salud nieguen la autorización de cirugía por laparoscopia es el temor de un número elevado de complicaciones. Sin embargo ya existe suficiente experiencia acumulada que nos indica la seguridad de los procedimientos ginecológicos laparoscópicos cuando son realizados por grupos con experiencia. En este sentido destaca el estudio de Querleu et al (15) en más de 17.000 laparoscopias realizadas en siete centros de Francia, en un período de 4 años. En 8.343 laparoscopias diagnósticas y 9.187 operatorias, las tasas de complicaciones que resultaron en laparotomías no deseadas fueron las siguientes: $1.7 / 1000$ para laparoscopia Diagnóstica; 0.4/1000 para procedimientos menores (Adhesiolisis mínima, ablación endometriosis leve, biopsia o punción ovárica, esterilización tubárica y reproducción asistida); 4.8/1000 para procedimientos quirúrgicos mayores (Adherencias extensas, tuboplastia, suspensión uterina, embarazo ectópico, salpingitis y endometriosis moderada o severa); 8.9/1000 para ciru- gías avanzadas (Histerectomía, anexectomía, miomectomía, uretrocistopexia y linfadenectomía). Ocurrió una muerte en el grupo de cirugía mayor, ocasionada por trauma con trocar a un gran vaso. Esta frecuencia de lesión vascular mayor concuerda con la cifra aproximada de un caso por cada 20.000 laparoscopias (16).

La mortalidad reportada está entre 4 a 8 pacientes por cada 100.000 laparoscopias (de todas las clases) $(16,17)$. Vale la pena anotar que en Estados Unidos hasta un tercio de las muertes asociadas con laparoscopias se ha atribuido a complicaciones anestésicas, especialmente con anestesia general sin intubación de las pacientes (16).

\section{Conclusiones}

En síntesis, creo razonable concluir que actualmente en Colombia la primera opción quirúrgica para la mayoría de la patología ginecológica benigna intraabdominal, debe ser la Laparoscopia ginecológica operatoria, siempre y cuando se pueda realizar en condiciones adecuadas $(13,14)$, y de manera ambulatoria. Esto se explica por las ventajas que representa para la paciente el acortamiento de la incapacidad, la baja frecuencia de complicaciones (con ginecólogos experimentados y certificados en este tipo de cirugía); por la disminución en costos globales para cada intervención, y porque al convertir un gran número de procedimientos que antes requerían laparotomía en cirugía ambulatoria, permite un manejo más racional de las camas hospitalarias para patologías y cirugías que sí ameritan hospitalización.

\section{BIBLIOGRAFIA}

1. Broussard T/, Mckernan J/, Laws H. Horizons in Endoscopic Surgery. En: Braverman M., Tawes R., eds. Surgical Technology International II. $1^{\mathrm{a}}$. ed. San Francisco, California. Editado por Surgical Technology International 1993; 37-39

2. Nava R., González E. Indicaciones, contraindicaciones y limitaciones de la cirugía laparoscópica. Ventajas y desventajas. En: Nava R./ Molina A., eds. Endoscopia Quirúrgica Ginecológica. 1ª. Ed. México: Mcgraw-Hill Interamericana Editores S.A.; 1997: 11-15.

3. Future Directions. En: Gomel V., Taylor P., eds. Diagnostic and Operative Gynecologic Laparoscopy. St. Louis, Missouri: MosbyYear Book, inc.; 1995: 310-312.

4. Instituto de Seguros Sociales. Acuerdo número 209 de 1.999. Diario Oficial No. 43567, Abril 30, 1999.

5. Ministerio de Salud. Manual de Tarifas y Procedimientos. Decreto 2423 del 31 de Diciembre de 1996. (S.O.A.T.).

6. Relative Value Units and Current Procedural terminology Codes. En: Hulka J., Reich H., eds. Textbook o Laparoscopy. 3rd. Ed. New York, New York: W. B. Saunders Company; 1998: 525-527.

7. Azziz R. Advantages and Disadvantages of Operative Endoscopy. En: Azziz R., Alvarez A., eds. Practical Manual of Operative Laparoscopy and Histeroscopy. Springer Verlag. New York, inc. 1992: 1-6.

8. Abascal A., Pérez A., Méndez F. Estudio de costo beneficio, costo eficiencia y costo financiero de la cirugía endoscópica. En: Nava R., Molina A., eds. Endoscopia Quirúrgica Ginecológica. $1^{a}$. Ed. México: Mcgraw-Hill Interamericana Editores S.A.; 1997: 58-60.
9. Johns A. Cost Effectiveness and Laparoscopic Surgery. En Adamson D., Martin D., eds. Endoscopic Management of Gynecologic Disease, Philadelphia, Pennsylvania: Lippincort-Raven Publishers; 1996: 415419

10. Medina J. Historia de los comienzos de la laparoscopia en Colombia. Rev. Col. Ginecol.1.997; 48: 75-78.

11. Patiño J. et al. La colecistectomía Laparoscópica. Triv. Med. 1992;85 (6): 237-246.

12. Hulka J., Reich H. Laparoscopic Histerectomy. En: Hulka J, Reich H., eds. Textbook of Laparoscopy. 3rd. Ed. New York, New York: W. B. Saunders Company; 1998: 363-379.

13. Manual de Reglamentación en Endoscopia Ginecológica. Sociedad Colombiana de Obstetricia y Ginecología. Santafe de Bogotá: 1997.

14. Argüello R. Entrenamiento y certificación en endoscopia ginecológica. Rev. Col. Ginecol. 1998; 49: 111-114.

15. Querleu D., Chevallier L., Chapron C., Bruhat M. Complication of laparoscopy surgery. A French multicentre collaborative study. Gynaecol. Endosc.1993; 2: 3-6.

16. Survey Data: Mortality and Morbidity. En Hulka J, Reich H., eds. Textbook of Laparoscopy. 3rd. Ed. New York, New York: W. B. Saunders Company; 1998: 505-511.

17. Newton M. Complications of abdominal operations. En Newton M., Newton E., eds. Complications of Gynecologic and Obstetric Management. Philadelphia, Pennsylvania: W. B. Saunders Company; 1998: $143-174$ 